\title{
Vector dark matter and Fermi-LAT gamma ray line
}

\section{Ki-Young Choi}

Asia Pacific Center for Theoretical Physics, Pohang, Gyeongbuk 790-784, Republic of Korea and Department of Physics, POSTECH, Pohang, Gyeongbuk 790-784, Republic of Korea

E-mail: kiyoung.choi@apctp.org

\section{Hyun Min Lee}

School of Physics, KIAS, Seoul 130-722, Republic of Korea

E-mail: hyun.min.leedkias.re.kr

\section{Osamu Seto*}

Department of Life Science and Technology, Hokkai-Gakuen University, Sapporo 062-8605,

Japan

E-mail: seto@physics.umn.edu

\begin{abstract}
We propose a renormalizable model for vector dark matter with an extra $U(1)$ gauge symmetry broken by a complex singlet scalar. When the singlet scalar has a quartic coupling to a heavy charged scalar, the resonance effect in s-channel singlet-like scalar exchange processes relatively enhances the annihilation cross section of vector dark matter into two photons such that Fermigamma ray line at about $130 \mathrm{GeV}$ can be explained. Due to the tiny mixing between the singlet scalar and the Standard Model Higgs doublet, the correct thermal relic density is also obtained by dominantly the annihilation into $W W / Z Z$ as well as $\gamma \gamma$.
\end{abstract}

The European Physical Society Conference on High Energy Physics -EPS-HEP2013

18-24 July 2013

Stockholm, Sweden

* Speaker. 


\section{Introduction}

Recently, the line feature of spectrum at $E_{\gamma}=130 \mathrm{GeV}$ has been claimed in Fermi-LAT gamma ray data $[1,2]$. The signature has been independently confirmed by other groups $[3,4]$. The FermiLAT collaboration [5] has reported a less significant peak at $E_{\gamma}=133 \mathrm{GeV}$ than that in Ref. [2]. Although there are some possible explanations such as mono-energetic pulsar winds [6], Fermi Bubble $[7,8]$ or instrumental effects $[9,10,11]$ including Earth limb signal $[5,12]$, the dark matter interpretation of the Fermi-LAT line signature seems plausible.

Dark matter is neutral and thus cannot annihilate into photons at tree-level. The generation of photons must happen via the loops of charged particles to which dark matter is directly or indirectly coupled, so the annihilation cross section of dark matter into photons is much suppressed as compared to other tree-level annihilation channels. Thus, in order to realize a large branching fraction of the annihilation cross section into photons, we need to rely on a large coupling to new charged particles running in loops $[13,14,15]$ or a resonance pole of the mediator particle between dark matter and photons $[16,17]$.

The extra $U(1)$ gauge symmetry is one of the simplest extensions of the standard model (SM). In this paper, we propose a renormalizable model of vector dark matter where the extra $U(1)_{X}$ gauge boson is a dark matter candidate and couples to the SM particles through the mixing between the $U(1)_{X}$ breaking scalar and the SM Higgs doublet [18]. When there is a quartic coupling between the singlet scalar and a heavy charged scalar, dark matter can annihilate into a photon pair with sizable branching fraction, provided that the mixing between the singlet scalar and the SM Higgs boson is small enough. The annihilation cross section of dark matter into a photon pair is enhanced near the resonance pole of the singlet-like scalar mediator to be consistent with Fermi gamma-ray line. For a tiny mixing between the neutral scalars, the thermal relic density can be determined dominantly by annihilation channels into $W W, Z Z$ and a photon pair.

We note that in most of other previous works on vector WIMP, a vector boson was introduced as a Proca field $[19,20,21]$ or a scalar sector is described by the nonlinear sigma model [22]. In our model, vector dark matter is realized as a gauge boson of extra $U(1)$ symmetry when it is broken spontaneously by the VEV of the hidden Higgs field at the renormalizable level [20, 23].

\section{The model}

We consider a simple model of vector WIMP dark matter which couples to the SM particles through Higgs portal interactions. The gauge sector of the model is based on the $S U(3)_{C} \times$ $S U(2)_{L} \times U(1)_{Y} \times U(1)_{X}$ gauge group. The extra $U(1)_{X}$ gauge symmetry is spontaneously broken by the vacuum expectation value (VEV) of a complex scalar $S_{1}$. For a minimal extension of the SM, we introduce an $S U(2)$ singlet charged scalar $S_{2}^{+}$, which carries $Y=1$ but is neutral under $U(1)_{X}$. We assume that all the SM particles including the Higgs doublet are neutral under the $U(1)_{X}$.

The model has a $Z_{2}$ symmetry under which $S_{1} \rightarrow S_{1}^{*}$ and $X_{\mu} \rightarrow-X_{\mu}$, which guarantees the stability of vector dark matter $X_{\mu}$. The Lagrangian of the model is

$$
\mathscr{L}=-\frac{1}{4} F_{\mu v} F^{\mu v}+\left|D_{\mu} S_{1}\right|^{2}+\left|D_{\mu} S_{2}\right|^{2}-V\left(\Phi, S_{1}, S_{2}\right)+f_{i j} L_{i} C \cdot L_{j} S_{2}^{+},
$$



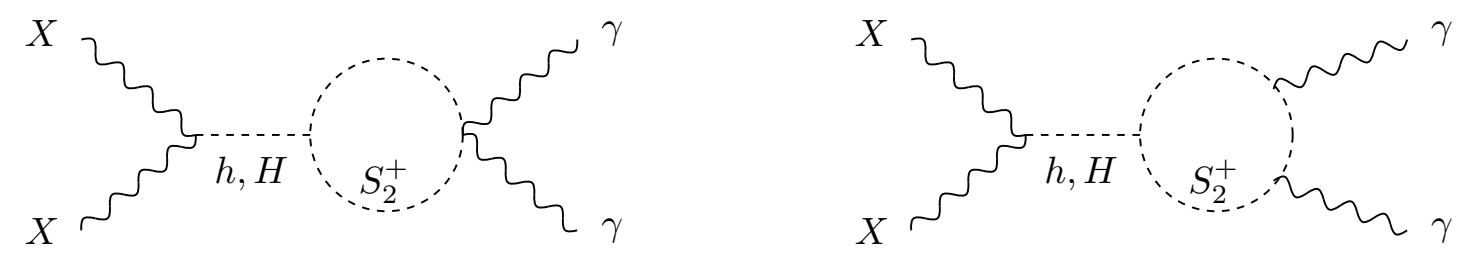

Figure 1: Feynman diagrams for the annihilation of vector dark matter into a photon pair at one-loop.

where $F_{\mu \nu}=\partial_{\mu} X_{v}-\partial_{v} X_{\mu}$, and the covariant derivatives are $D_{\mu} S_{1}=\left(\partial_{\mu}-i g_{X} X_{\mu}\right) S_{1}$ and $D_{\mu} S_{2}=$ $\left(\partial_{\mu}-i g^{\prime} B_{\mu}\right) S_{2}$, with respect to $U(1)_{X}$ and $U(1)_{Y}$ gauge symmetry, respectively. After the electroweak symmetry breaking, $D_{\mu} S_{2}$ is reduced to the covariant derivative with respect to $U(1)_{\mathrm{em}}$ symmetry. The scalar potential of the SM Higgs doublet $\Phi$ and complex scalar fields $S_{1}$ and $S_{2}$ is given by

$$
\begin{aligned}
V\left(\Phi, S_{1}, S_{2}\right)= & \mu_{1}^{2}|\Phi|^{2}+\mu_{2}^{2}\left|S_{1}\right|^{2}+\mu_{3}^{2}\left|S_{2}\right|^{2}+\frac{1}{2} \lambda_{1}|\Phi|^{4}+\frac{1}{2} \lambda_{2}\left|S_{1}\right|^{4}+\frac{1}{2} \lambda_{3}\left|S_{2}\right|^{4} \\
& +\lambda_{4}|\Phi|^{2}\left|S_{1}\right|^{2}+\lambda_{5}|\Phi|^{2}\left|S_{2}\right|^{2}+\lambda_{6}\left|S_{1}\right|^{2}\left|S_{2}\right|^{2}
\end{aligned}
$$

Here the $\lambda_{4}$ coupling is relevant for the mixing between $\Phi$ and $S_{1}$, after the breaking of $U(1)_{X}$ and electroweak symmetry. The dominant annihilation of vector dark matter occurs through this coupling so that the correct relic density can obtained. The coupling $\lambda_{6}$ connects the vector dark matter to the charged particle and enhances the photon emission. In the last term of the Lagrangian (2.1), $L_{i}$ is the SM lepton doublet with flavor index $i=1,2,3, C$ is the charge-conjugation operator and dot denotes the $S U(2)$ anti-symmetric product. This term induces the decay of heavy charged scalar $S_{2}^{ \pm} \rightarrow l_{i}^{ \pm}+\bar{v}_{j}$.

At the vacuum with a nonvanishing singlet VEV, $\left\langle S_{1}\right\rangle=v_{S} / \sqrt{2}$, the $U(1)_{X}$ gauge symmetry is broken so the gauge boson $X_{\mu}$ acquires mass $M_{X}^{2}=g_{X}^{2} v_{S}^{2}$. At the true vacuum with a nonvanishing $v_{S},\langle\Phi\rangle=v / \sqrt{2}$ and $v \simeq 246 \mathrm{GeV}$, two scalar modes appear from $\Phi$ and $S$ and mix in the mass eigenstates $h$ and $H$. The Higgs mixing angle essentially depends on $\lambda_{4}$ through $\tan 2 \alpha=\frac{2 \lambda_{4} v v_{S}}{\lambda_{1} v^{2}-\lambda_{2} v_{S}^{2}}$. For a small mixing angle $\alpha$, we can regard $h$ as being SM Higgs-like and $H$ as being singlet-like.

\section{Relic density and Fermi gamma-ray lines}

In this section, we compute the DM annihilation cross sections and discuss the constraints of the relic density and the Fermi gamma-ray line in the model.

\subsection{Thermal relic abundance}

The thermal relic abundance of the vector WIMP dark matter $X$ is estimated by integrating the following Boltzmann equation for the dark matter number density $n_{X}$ in the early Universe.

$X$ dominantly annihilates into $W$ and $Z$ boson pairs through the s-channel exchange of the Higgs bosons $h$ and $H$. The magnitude of those annihilation cross sections is proportional to $(\sin \alpha \cos \alpha)^{2}$ and thus scaled by the mixing angle between Higgs bosons. A larger (smaller) annihilation cross section is realized for a larger (smaller) $\sin \alpha$. With a sizable quartic coupling between the singlet scalar $S_{1}$ and the heavy charged scalar $S_{2}$, dark matter can annihilate into a photon pair, due to loops 
with the charged scalar $S_{2}^{+}$as shown in Fig. 1. This process induces a large enough annihilation cross section into two gammas to explain the line feature, as will be shown in the next subsection.

We are interested in the case where $X$ has a large annihilation cross section into two photons so that the gamma ray line at $135 \mathrm{GeV}$ observed by Fermi LAT can be explained. Taking the annihilation into a $W$ or $Z$ boson pair through $h$ and $H$-exchange to be strongly suppressed due to a tiny mixing angle between SM Higgs boson and singlet scalars, namely, $|\sin \alpha| \ll 1$, we obtain the desired thermal relic density and the necessary cross section into a photon pair for Fermi gammaray line near the resonance pole of the singlet-like scalar $[16,17]$. Therefore, near the resonance, $M_{X} \simeq M_{H} / 2$, for the WIMP mass ${ }^{1}$ around $135 \mathrm{GeV}$, we need to take the singlet-scalar mass $M_{H}$ to be about $270 \mathrm{GeV}$. In Fig. 2, we show that the right thermal relic density of WIMP can be realized with appropriate set of parameters.

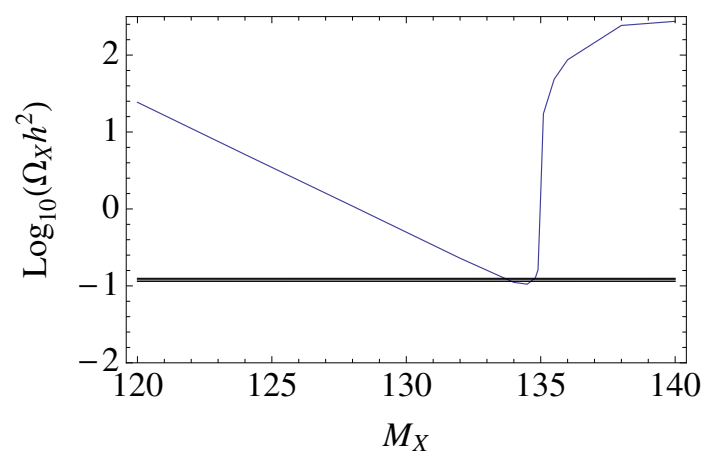

Figure 2: The plot of relic density of the dark matter $\Omega h^{2}$ vs. its mass. Here we used $M_{S_{2}^{ \pm}}=140 \mathrm{GeV}$, $v_{S}=1 \mathrm{TeV}, \sin \alpha=0.00047$ and $\lambda_{6}=0.2$. We can ignore the $\lambda_{5}$ dependence for the parameter region in our interest. The horizontal line is the relic density of cold dark matter revealed by the Planck result, $\Omega_{\mathrm{CDM}} h^{2}=0.1199 \pm 0.0027$ [24].

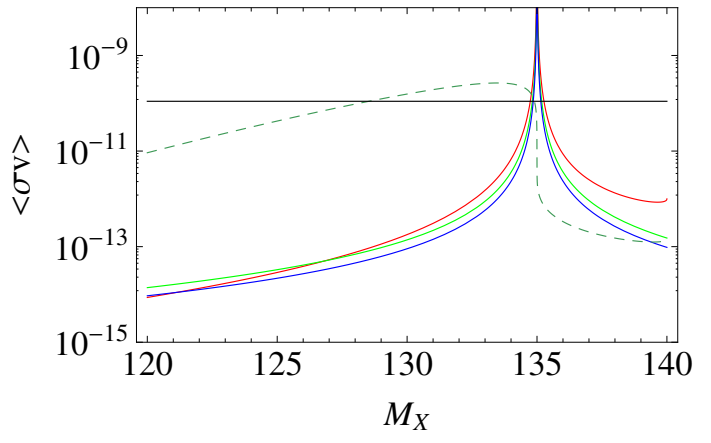

Figure 3: The dashed line is the total thermal averaged annihilation cross section at the moment of freeze-out. Solid lines are annihilation cross sections for each mode at present (Red: $\gamma \gamma$, Blue: $W W$, Green: $Z Z$ final states). The horizontal black line represents the DM annihilation cross section into two photons $\langle\sigma v\rangle_{\chi \chi \rightarrow \gamma \gamma}$ needed for the Fermi-LAT gamma ray line.

\subsection{Monochromatic photons from DM annihilation}

Now we discuss the aspect of the indirect detection of vector WIMP. In addition to the $W W / Z Z$ annihilation channels, $X$ can also annihilate into two monochromatic photons with the effective interaction induced by the heavy charged scalar. Hence, this diphoton mode can provide a source for the gamma-ray line observed by Fermi LAT. When the singlet-like scalar $H$ has small couplings to $W$ and $Z$ bosons due to a tiny $\sin \alpha$ but it has a sizable coupling to the charged scalar $S_{2}$, the diphoton mode takes a larger branching fraction of the annihilation cross section than usually expected.

Keeping only the $H$-exchange contribution for a small Higgs mixing and using $s \simeq 4 M_{X}^{2}$, we get an approximate form for the thermal averaged annihilation cross section into a photon pair at

\footnotetext{
${ }^{1}$ Of course, there is another resonance region $M_{X} \simeq M_{h} / 2 \simeq 63 \mathrm{GeV}$ where the main annihilation mode is $X X \rightarrow b \bar{b}$.
} 
present as

$$
\langle\sigma v\rangle_{\gamma \gamma} \simeq \frac{\alpha_{\mathrm{em}}^{2}}{96 \pi^{3} M_{X}^{2}}\left|\frac{\lambda_{6} \cos ^{2} \alpha M_{X}^{2}}{4 M_{X}^{2}-M_{H}^{2}+i M_{H} \Gamma_{H}}\right|^{2}\left|1-\frac{M_{S_{2}^{+}}^{2}}{M_{X}^{2}} f\left(\frac{M_{S_{2}^{+}}^{2}}{M_{X}^{2}}\right)\right|^{2},
$$

with $f(\tau)=\left[\sin ^{-1}(1 / \sqrt{\tau})\right]^{2}[25]$. Figure. 3 shows the annihilation cross sections into $W W, Z Z, \gamma \gamma$ at zero temperature, and the thermal averaged total annihilation cross section used to estimate $\Omega h^{2}$, respectively. To explain the gamma ray line spectrum of the Fermi-LAT for the Einasto dark matter profile [1, 2], we require that $\langle\sigma v\rangle_{\chi \chi \rightarrow \gamma \gamma}=\left(1.27 \pm 0.32_{-0.28}^{+0.18}\right) \times 10^{-27} \mathrm{~cm}^{3} \mathrm{~s}^{-1} \simeq 1.1 \times$ $10^{-10} \mathrm{GeV}^{-2}$.

In order to obtain the observed Fermi gamma-ray line together with the correct relic density, the small mixing angle, $|\sin \alpha| \ll 1$, is necessary. This small $\sin \alpha$ is important to suppress annihilations into $W W$ and $Z Z$ not to generate too many continuum photons as well [26, 27, 28].

The same diagrams in Fig. 1 applies to the annihilation of vector dark matter into $Z \gamma$ and loop induced $Z Z$ final states. The annihilation $X X \rightarrow Z \gamma$ emits additional gamma line at $E_{\gamma}=114 \mathrm{GeV}$ and the resulting flux is suppressed by 0.21 as compared to that of $130 \mathrm{GeV}$ gamma ray line [14], while the loop induced annihilation into $Z Z$ is negligible.

\section{Conclusion}

We have proposed a renormalizable model of vector dark matter where the extra $U(1)_{X}$ gauge boson is a dark matter candidate and it interacts with the SM particles through the Higgs portal term, namely, the mixing between the $U(1)_{X}$ breaking singlet scalar and the SM Higgs doublet. If the Higgs mixing is small enough, the DM annihilations into $W$ and $Z$ boson pairs at the resonance pole of the singlet-like scalar can reproduce the correct thermal relic density without overproducing continuum photons. In the presence of a quartic coupling between the singlet scalar and the charged scalar $S_{2}$, vector dark matter also annihilates into a photon pair with a sizable branching fraction at the same singlet resonance.

\section{Acknowledgments}

K.-Y.C was supported by Basic Science Research Program through the National Research Foundation of Korea (NRF) funded by the Ministry of Education, Science and Technology (No. 2011-0011083). K.-Y.C acknowledges the Max Planck Society (MPG), the Korea Ministry of Education, Science and Technology (MEST), Gyeongsangbuk-Do and Pohang City for the support of the Independent Junior Research Group at the Asia Pacific Center for Theoretical Physics (APCTP).

\section{References}

[1] T. Bringmann, X. Huang, A. Ibarra, S. Vogl and C. Weniger, JCAP 1207, 054 (2012).

[2] C. Weniger, JCAP 1208, 007 (2012).

[3] E. Tempel, A. Hektor and M. Raidal, JCAP 1209, 032 (2012) [Addendum-ibid. 1211, A01 (2012)]. 
[4] M. Su and D. P. Finkbeiner, arXiv:1206.1616 [astro-ph.HE].

[5] [Fermi-LAT Collaboration], Phys. Rev. D 88, 082002 (2013).

[6] F. Aharonian, D. Khangulyan and D. Malyshev, arXiv:1207.0458 [astro-ph.HE].

[7] M. Su, T. R. Slatyer and D. P. Finkbeiner, Astrophys. J. 724, 1044 (2010).

[8] S. Profumo and T. Linden, JCAP 1207, 011 (2012).

[9] D. Whiteson, JCAP 1211, 008 (2012).

[10] D. P. Finkbeiner, M. Su and C. Weniger, JCAP 1301, 029 (2013).

[11] D. Whiteson, Phys. Rev. D 88, 023530 (2013).

[12] A. Hektor, M. Raidal and E. Tempel, Eur. Phys. J. C 73, 2578 (2013).

[13] J. M. Cline, Phys. Rev. D 86, 015016 (2012).

[14] K. -Y. Choi and O. Seto, Phys. Rev. D 86, 043515 (2012) [Erratum-ibid. D 86, 089904 (2012)].

[15] S. Tulin, H. -B. Yu and K. M. Zurek, Phys. Rev. D 87, 036011 (2013).

[16] H. M. Lee, M. Park and W. -I. Park, Phys. Rev. D 86, 103502 (2012); H. M. Lee, M. Park and W. -I. Park, JHEP 1212, 037 (2012); H. M. Lee, M. Park and V. Sanz, JHEP 1303, 052 (2013); D. Das, U. Ellwanger and P. Mitropoulos, JCAP 1208, 003 (2012); K. Schmidt-Hoberg, F. Staub and M. W. Winkler, JHEP 1301, 124 (2013).

[17] E. Dudas, Y. Mambrini, S. Pokorski and A. Romagnoni, JHEP 0908014 (2009); Y. Mambrini, JCAP 0912005 (2009); C. B. Jackson, G. Servant, G. Shaughnessy, T. M. P. Tait and M. Taoso, JCAP 1004 004 (2010); E. Dudas, Y. Mambrini, S. Pokorski and A. Romagnoni, JHEP 1210123 (2012).

[18] K. -Y. Choi, H. M. Lee and O. Seto, Phys. Rev. D 87, 123541 (2013).

[19] S. Kanemura, S. Matsumoto, T. Nabeshima and N. Okada, Phys. Rev. D 82, 055026 (2010).

[20] O. Lebedev, H. M. Lee and Y. Mambrini, Phys. Lett. B 707, 570 (2012); A. Djouadi, O. Lebedev, Y. Mambrini and J. Quevillon, Phys. Lett. B 709, 65 (2012).

[21] Y. Farzan and A. R. Akbarieh, arXiv:1211.4685 [hep-ph].

[22] T. Abe, M. Kakizaki, S. Matsumoto and O. Seto, Phys. Lett. B 713, 211 (2012).

[23] S. Baek, P. Ko, W. -I. Park and E. Senaha, JHEP 1305, 036 (2013).

[24] P. A. R. Ade et al. [ Planck Collaboration], arXiv:1303.5076 [astro-ph.CO].

[25] J. F. Gunion, H. E. Haber, G. Kane, and S. Dawson, The Higgs Hunter's Guide, Addison-Wesley (1990).

[26] W. Buchmuller and M. Garny, JCAP 1208, 035 (2012).

[27] T. Cohen, M. Lisanti, T. R. Slatyer and J. G. Wacker, JHEP 1210, 134 (2012).

[28] M. Asano, T. Bringmann, G. Sigl and M. Vollmann, Phys. Rev. D 87, 103509 (2013). 